\title{
ERECTOR SPINAE PLANE BLOCK (ESPb) FOR AWAKE BREAST SURGERY IN YOUNG PREGNANT PATIENT. A CASE REPORT
}

\author{
Luca Aiello, Claudio Pitrè, Daniele Bellantonio, Alberto Gori, Vinicio Dima, Ruggero \\ Massimo Corso, Stefano Maitan.
}

Correspondence: aielluca@gmail.com; Dipartimento Chirurgico, Ospedale Morgagni-Pierantoni, Forli, Via Carlo Forlanini, 34,47121

Forli (FC) - Italy; phone number +393283144897

Background and aims: ESPb was described in thoracic neuropathic pain ${ }^{(1)}$.It is not clear data over its analgesic range.ESPb prevents visceral and somatic pain including breast surgery(2). Here, we present a successful ESPb in awake anaesthesia for radical mastectomy.

Methods: a 31year old pregnant patient was scheduled for nipple-areola complex sparing total mastectomy with axillary clearance of lymph nodes and expander positioning.Cardiotocographic control was carried out before and after surgery.ESPb was performed in sitting position at T4 transverse process level using $10-\mathrm{MHz}$ linear ultrasound probe.Ropivacaine $0.5 \%, 20 \mathrm{ml}$ was used.After confirming that successful blockade from T2-T8 was achieved in $30 \mathrm{~min}$, propofol (target effect site concentration; $1 \mu \mathrm{g} / \mathrm{ml}$ ) was infused to achieve a Ramsay sedation score of 4 .No opioids were needed throughout surgery that lasted about 180 minutes.

Results: her vital signs were stable during surgery. The patient experienced very good analgesia and described NRS pain score 0-2 up to 18 hours after ESPb.After 18hours and only once in the next 12 hours, the patient requested "on demand" pain medication (Paracetamol $1 \mathrm{gr}$ ). The patient experienced no nausea or vomiting, and she was easily mobilized.

Conclusions: ESPb is a simple and safe block,avoid opioid use.Our experience demonstrates that ESPb with sedation is a suitable option for awake radical mastectomy and reduce risk of PONV.

\section{REFERENCES:}

1) M. Forero,S.D. Adhikary,H. Lopez.C. Tsui,K. J. Chin. The Erector Spinae Plane Block A Novel Analgesic Technique in Thoracic Neuropathic Pain.Regional Anesthesia and Pain Medicine,2016:41;5,1-7

2) Ohgoshi Y, Ikeda T,Kurahashi K.Continuous erector spinae plane block provides effective perioperative analgesia for breast reconstruction using tissue expanders:a report of two cases. JClinAnesth.2018;44:1-2
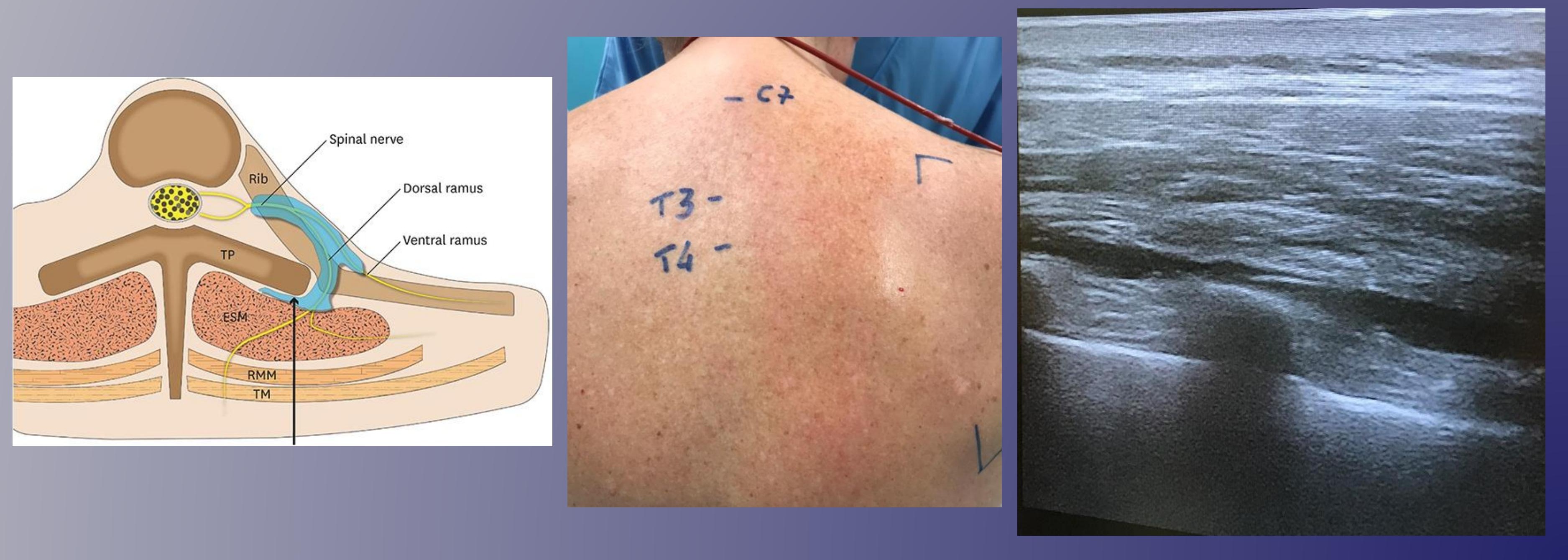